HIAS-E-48

\title{
Natural implementation with semi-responsible agents in pure exchange economies
}

\author{
Michele Lombardi \\ Adam Smith Business School, University of Glasgow, Glasgow, G12 8QQ, United Kingdom \\ Naoki Yoshihara \\ University of Massachusetts Amherst, Crotty Hall, 412 North Pleasant Street, Amherst, MA 01002, USA \\ The Institute of Economic Research, Hitotsubashi University, Naka 2-1, Kuni- tachi, Tokyo 186-8603, Japan \\ School of Management, Kochi University of Technology, Tosayamada, Kami-city, Kochi 782-8502, Japan
}

December 2016

Hitotsubashi Institute for Advanced Study, Hitotsubashi University

2-1, Naka, Kunitachi, Tokyo 186-8601, Japan

tel:+81 $425808604 \quad$ http://hias.ad.hit-u.ac.jp/

HIAS discussion papers can be downloaded without charge from:

http://hdl.handle.net/10086/27202

https://ideas.repec.org/s/hit/hiasdp.html

All rights reserved. 


\title{
Natural implementation with semi-responsible agents in pure exchange economies*
}

\author{
Michele Lombardi ${ }^{\dagger}$ and Naoki Yoshihara ${ }^{\ddagger}$
}

December 12, 2016

We study Nash implementation by natural price-quantity mechanisms in pure exchange economies when agents have intrinsic preferences for responsibility. An agent has an intrinsic preference for responsibility if she cares about truth-telling that is in line with the goal of the mechanism designer besides her material well-being. A semi-responsible agent is an agent who, given what her opponents do, acts in an irresponsible manner when a responsible behavior poses obstacles to her material well-being. The class of efficient allocation rules that are Nash implementable is identified provided that there is at least one agent who is semi-responsible. The Walrasian rule is shown to belong to that class.

JEL classification: C72; D71.

Key-words: Nash equilibrium, exchange economies, intrinsic preferences for responsibility, boundary problem, price-quantity mechanism.

\section{Introduction}

In implementation problems in pure exchange markets, as Hurwicz (1978), Otani and Sicilian (1982) and Thomson (1984) discuss, the information exchanged in the market typically concerns prices of commodities and the agents' consumption bundles, subsequently quantities, at those prices. Therefore, a prominent restriction on mechanisms for implementation problems in pure exchange markets would be represented by price-quantity mechanisms, henceforth $p-q$ mechanisms: each agent chooses a price vector $p$ as well as a quantity $q$ as her strategy choice (see also Hurwicz et al., 1995).

*We are grateful to an associate editor and two referees of this Journal for their useful comments and suggestions. They are not responsible for remaining deficiencies.

${ }^{\dagger}$ Adam Smith Business School, University of Glasgow, Glasgow, G12 8QQ, United Kingdom, e-mail: lombardimichele1975@gmail.com.

${ }^{\ddagger}$ Department of Economics, University of Massachusetts Amherst, 200 Hicks Way, Amherst, MA 01003, USA; The Institute of Economic Research, Hitotsubashi University, Kunitachi, Tokyo 1860004 Japan; and School of Management, Kochi University of Technology, Kochi 782-8502, Japan. E-mail: n_yoshihara_1967@yahoo.co.jp 
To meet the requirements for an adequate theory of implementation (see as per Jackson, 1992), Dutta et al. (1995), Sjöström (1996), and Saijo et al. (1996, 1999) have defined the class of natural mechanisms in economic environments. Within this class, mechanisms are typically required $a$ ) to ensure each agent always receives a quantity lying in her consumption set; $b$ ) to ensure each agent receives, in equilibrium, what she stated as her own quantity; and $c$ ) to ensure each agent has a best strategy choice for every other agents' strategy choices. ${ }^{1}$ We then name any $p-q$ mechanism that meets these requirements a natural $p$ - $q$ mechanism.

Matsushima (2008a, 2008b) and Dutta and Sen (2012) introduced and studied implementation problems when agents are partially-honest. In a strategic game, an agent is partially-honest if she tells the truth when truth-telling results in the highest material payoff available to her given other agents' strategy choices. ${ }^{2}$ Simply put, as between being truthful and being untruthful, a partially-honest agent prefers to be truthful when that choice of behavior does not hurt her material well-being given her opponents' actions. In a general environment, Dutta and Sen (2012) show that for implementation problems involving at least three individuals and in which there is at least one partially-honest individual, the Nash implementability is assured by no veto-power.

This line of research is particularly relevant if the task at hand is to achieve social goals via market like-mechanisms. Indeed, the analysis of this class of implementation problems typically relies on the assumption that maximization of material gains is the only intention of agents' actions. There is therefore a need to develop decentralized decision making processes that can take into account more sophisticated behavior than the one that has been analyzed up until this point. This paper studies the effect of responsible actions in the design of market like-mechanisms. In fact, simple reasoning and everyday observation suggest that a concern about responsibility as well as welfare of the society is an important determinant of behavior. Actual behavior is often the outcome of a compromise between what society prescribes and what the pursuit of material gains dictates.

We adopt herein the common view in the literature of resource allocation mechanisms that an agent is responsible when she states the true prices and demands an affordable utility-maximizing quantity at those prices and when her statement fosters the pursuit of socially valued goals. As an example, think of the Walrasian equilibrium: an agent is responsible if she reports competitive equilibrium prices as well as a competitive equilibrium quantity at those prices. In line with the notion of partially-honest agent introduced by Dutta and Sen (2012), we say that an agent is semi-responsible if she complies with the welfare criterion incorporated into allocation rule in the absence of opportunistic behavior. Simply put, a semi-responsible agent dislikes acting in an irresponsibly opportunistic manner when a responsible conduct is equally efficacious. ${ }^{3}$

1. These requirements are formally defined in section 3 below.

2. There are seminal related works such as Glazer and Rubinstein (1998), Eliaz (2002), Corchón and Herrero (2004) and Kartik and Tercieux (2012).

3. Doğan (2014) studies problems of the fair allocation of indivisible goods by employing a similar 
On this basis, the paper develops a Nash implementation framework with semiresponsible agents where Nash implementation problems in pure exchange economies are studied, the class of efficient allocation rules that are Nash implementable by natural $p-q$ mechanisms is identified and, finally, shows that the Walrasian rule belongs to this class.

The closest papers to ours are Dutta and Sen (2012) and Kartik et al. (2014), which are works on implementation with partially-honest agents in separable environments (as per Jackson, 2001). By focussing on social choice functions, these scholars establish general positive results by devising mechanisms that do not use any sort of "integer games" or "modulo games". Specifically, when every agent has an intrinsic preference for honesty, Dutta and Sen (2012) provide a separability condition under which any social choice function is implementable in strictly dominant strategies. ${ }^{4}$ When the mechanism designer knows the identity of at least one partially-honest agent or when every agent has an intrinsic preference for honesty, Kartik et al. (2014) offer a condition called separable punishment under which any social choice function is implementable by a simple mechanism via two rounds of iterated deletion of strictly dominated strategies. The devised mechanisms share a feature which does not qualify them as a market mechanism: Each agent's strategy choice includes at least her preference.

By contrast, we endorse a weaker, and what we consider to be a more reasonable, informational requirement for the mechanism designer: The designer only knows there exists at least one semi-responsible agent in the population. More importantly, our agents do not announce preferences as their strategy choices. In fact, by stating prices and consumption bundles, an agent transmits simply local information about her preferences to the mechanism designer. We also abandon the common requirement of the single-valuedness of allocation rules because it is incompatible, for example, with the property of many interesting allocation rules such as the multiplicity of Walrasian equilibria. Finally, like Dutta and Sen's (2012) and Kartik et al.'s (2014) results, our characterization result does not rely on any sort of "tail-chasing" construction.

It is well-known by Hurwicz et al. (1995) that the Walrasian rule does not satisfy Maskin monotonicity, a necessary condition for Nash implementation. The reason is that the Walrasian rule also depends on the shape of preferences outside of the set of feasible allocations. This problem is known as boundary problem and has stimulated a stream of informative studies.

The way around this problem suggested by Hurwicz et al. (1995) is to focus on the constrained Walrasian rule, which is the minimal monotonic extension of the Walrasian rule (Thomson, 1999). ${ }^{5}$ Postlewaite and Wettstein (1989), Dutta et al. (1995), Saijo et al. (1996) and Tian (1992) show that the constrained Walrasian rule is implementable by a price-allocation mechanism: each agent chooses a price vector as well as an allocation as her strategy choice. Saijo et al. (1996) also show that the constrained

definition of responsible agents.

4. This informational assumption is used also in other environments; see, e.g., Saporiti (2014).

5. The constrained Walrasian rule is a super-correspondence of the Walrasian rule. The two rules coincide when agents' preferences are convex and Walrasian equilibrium allocations are interior. 
Walrasian rule is implementable by a $p-q$ mechanism provided that allocations on the boundary of the feasible set are ruled out. Saijo et al. (1999) focus on the boundary problem and show that the constrained Walrasian rule cannot be implemented by any natural $p-q$ mechanism in economies with more than two commodities. Lately, Bochet (2007) provides a thorough study of the boundary problem and shows that the Walrasian rule cannot be implemented in any of the solution concepts considered in the implementation literature if Walrasian equilibrium allocations on the boundary of the feasible set are not ruled out. This paper also takes care of the boundary problem that is prominent in Nash implementation literature and shows that in the case where there are at least three agents, the Walrasian rule is Nash implementable by a natural $p-q$ mechanism when at least one agent is semi-responsible and the mechanism designer does not know the identity of the semi-responsible agent(s). It is worth mentioning that, with respect to Saijo et al. (1999), this paper is built on a definition of natural $p-q$ mechanisms that differs from that in Saijo et al. (1999). This is so because we employ a definition of supporting prices that possibly also depend on the efficient allocation rule considered (to be discussed in section 3.1). This choice is dictated by the consideration that supporting prices are not equally important to every efficient allocation rule (as per Saijo et al., 1999).

Section 2 and section 3 of this paper present the theoretical framework and outline the basic model, with results presented in section 4. Section 5 concludes. Omitted proofs can be found in the Appendix.

\section{Preliminaries}

The framework can be described as follows. There are $n \geq 3$ agents and $\ell \geq 2$ commodities, where $n$ and $\ell$ are both finite. The set $N \equiv\{1, \ldots, n\}$ denotes the set of agents, and the set $L \equiv\{1, \ldots, \ell\}$ denotes the set of commodities.

Agent $i$ 's commodity bundle $x_{i}=\left(x_{i 1}, \ldots, x_{i \ell}\right)$ is an $\ell$-dimensional vector describing how much of each commodity agent $i$ consumes, where $x_{i l} \geq 0$ for all $l \in L$. The commodity space for agent $i$ is denoted by $\mathbb{R}_{+}^{\ell}$, where $\mathbb{R}_{+}^{\ell}$ is the non-negative orthant of the $\ell$-dimensional Euclidean space $\mathbb{R}^{\ell}$. An allocation $x=\left(x_{1}, \ldots, x_{n}\right) \in \mathbb{R}_{+}^{n \ell}$ is a profile of commodity bundles. We shall sometimes write $\left(x_{i}, x_{-i}\right)$ for the profile of commodity bundles, where $x_{-i}=\left(x_{1}, \ldots, x_{i-1}, x_{i+1}, \ldots, x_{n}\right)$; the same notational convention will be followed for any profile of objects.

We assume that each agent $i$ has an initial non-zero endowment of the $\ell$ commodities, $\omega_{i}=\left(\omega_{i 1}, \ldots, \omega_{i \ell}\right) \in \mathbb{R}_{+}^{\ell} \backslash\{0\}$. The endowment profile is denoted by $\omega=$ $\left(\omega_{1}, \ldots, \omega_{n}\right) \in \mathbb{R}_{+}^{n \ell}$. The aggregate endowment of commodity $l \in L$, denoted $\Omega_{l}$, is defined by $\Omega_{l} \equiv \sum_{i \in N} \omega_{i l}$ and assumed to be strictly positive. The aggregate endowment profile is denoted by $\Omega \equiv\left(\Omega_{1}, \ldots, \Omega_{\ell}\right)$.

We assume that each agent $i$ has a preference ordering over the commodity space $\mathbb{R}_{+}^{\ell}$, which is represented by a utility function $u_{i}$ that is continuous, quasi-concave and strongly monotonic on $\mathbb{R}_{+}^{\ell}$. The class of allowable continuous, quasi-concave and 
strongly monotonic utility functions for agent $i$ is denoted by $U_{i} \cdot{ }^{6}$

Since the set of agents, $N$, and the endowment profile, $\omega$, will remain fixed, an economic environment, subsequently environment, is described by a profile of utility functions $\left(u_{i}\right)_{i \in N}$, where $u_{i} \in U_{i}$ for every $i \in N$. The class of allowable environments is denoted by $U_{N}$ and defined by a subset of the Cartesian product $U_{1} \times \ldots \times U_{n}$.

A price vector is an $\ell$-dimensional vector $p=\left(p_{1}, \ldots, p_{\ell}\right)$, where the price of commodity $l \in L$ is $p_{l} \geq 0$. The value of a commodity bundle $x_{i}$ at prices $p$ is $p \cdot x_{i}$. Without loss of generality, we restrict the price space to the unit simplex in $\mathbb{R}^{\ell}$, denoted by $P$ and defined by the set of $p$ 's such that $\sum_{l \in L} p_{l}=1$ and $p_{l} \geq 0$ for any $l \in L$.

An allocation $x \in \mathbb{R}_{+}^{n \ell}$ is feasible if $\sum_{i \in N} x_{i} \leq \Omega .^{7}$ We denote by $A$ the set of (feasible) allocations. A commodity bundle $x_{i} \in \mathbb{R}_{+}^{\ell}$ is feasible if $x_{i} \leq \Omega$. We shall refer to a feasible commodity bundle $x_{i}$ as a quantity. The set of quantities is denoted by $Q$.

For any $u \in U_{N}$, an allocation $x \in A$ is (Pareto) efficient for $u$ if there is no other allocation $y \in A$ such that $u_{i}\left(y_{i}\right) \geq u_{i}\left(x_{i}\right)$ for all $i \in N$ with at least one of these inequalities being strict. We denote by $P O(u)$ the set of efficient allocations for $u$.

In this paper, we are interested in implementing efficient allocation rules. Formally:

Definition 1. An efficient allocation rule is a multi-valued (non-empty) mapping $F: U_{N} \rightarrow A$ such that for each $u \in U_{N}$ : (i) $F(u) \subseteq P O(u)$ and (ii) for each $x \in F(u)$ and $i \in N$, it holds that $x_{i}>0$. The class of efficient allocation rules is denoted by $\mathcal{F}$. We shall refer to $x \in F(u)$ as an $F$-allocation at $u$.

Given this notion, any efficient allocation rule in $\mathcal{F}$ ensures that every agent consumes a strictly positive amount of at least one commodity. As it will be evident, we use this requirement in the sufficiency part in order to be able to devise a natural $p-q$ mechanism that does not rely on any sort of "integer games" or "modulo games."

A (normal form) mechanism is a pair $\gamma \equiv(M, g)$, where $M \equiv M_{1} \times \ldots \times M_{n}, M_{i}$ is the strategy space of agent $i \in N$, and $g: M \rightarrow \mathbb{R}^{n \ell}$, the outcome function, assigns to every strategy profile $m \in M$ a unique allocation in $\mathbb{R}^{n \ell}$. Denote the $i$ th component of $g(m)$ by $g_{i}(m)$.

In this paper, we focus on price-quantity mechanisms: each agent announces a price vector, $p \in P$, and a quantity, $x_{i} \in Q$. Formally:

Definition 2. A mechanism $\gamma$ is a price-quantity mechanism, or simply a $p$ - $q$ mechanism, if for every $i \in N$, the strategy space of agent $i$ is $M_{i} \equiv P \times Q$, where $P$ is the price space and $Q$ is the set of quantities.

6. What we do in this paper extends to the case where $U_{i}$ is the class of allowable utility functions which are continuous and quasi-concave on $\mathbb{R}_{+}^{\ell}$, strongly monotonic on the strictly positive orthant of $\mathbb{R}^{\ell}$, and satisfy the following boundary condition: the utility of every interior commodity bundle is strictly higher than the utility of any boundary commodity bundle. The Cobb-Douglas utility function is an example of such utility functions.

7. We use the following conventions: For all $x, y \in \mathbb{R}_{+}^{\ell}, x \geq y$ if $x_{l} \geq y_{l}$ for each $l \in L ; x>y$ if $x \geq y$ and $x \neq y$; and $x \gg y$ if $x_{l}>y_{l}$ for each $l \in L$. 
Our interest in $p-q$ mechanisms stems from the fact that to implement Pareto efficient allocation rules in Nash equilibria we need to require agents to send some local information about their indifference curves, such as the common marginal rate of substitution at an efficient allocation, in addition to quantity announcements (Dutta et al., 1995; Saijo et al., 1996). The price announcement serves this purpose.

\section{Nash implementation by natural $p-q$ mechanisms with semi-responsible agents}

Below we provide the notion of Nash implementation by natural $p-q$ mechanisms when agents have intrinsic preferences for responsibility. We start by providing the definitions of supporting prices and of semi-responsible agents.

\subsection{Supporting prices}

As already noted, the price announcement is a device to obtain from agents information about the common marginal rate of substitution at an efficient allocation. If an efficient allocation rule generates only allocations in the interior of the feasible set $A$ and agents' utility functions are differentiable, the common marginal rate of substitution at any $F$-allocation is always unique. In these cases, a supporting price for $u$ at the efficient allocation $x$ can be taken to be the gradient vector of $u_{i}$ at the quantity $x_{i}$.

We lose, however, the uniqueness property of the marginal rates of substitution of agents since we neither assume differentiability of utility functions, nor the interiority of the range of allocation rules. In this case, one can define a supporting price for $u$ at $x$ as a price vector (or common subgradient) $p$ normal to a hyperplane separating the weak upper contour sets of any two utility functions in $u$ at the corresponding quantities in $x$. Formally:

Definition 3. (Saijo et al., 1999; p. 276) Let $u \in U_{N}$ and $x \in Q^{n}$ be given. The weak upper contour set of $u_{i}$ at $x_{i}$ is $V\left(x_{i}, u_{i}\right) \equiv\left\{x_{i}^{\prime} \in Q \mid u_{i}\left(x_{i}^{\prime}\right) \geq u_{i}\left(x_{i}\right)\right\}$. A price vector $p \in P$ is said to be a supporting price for $u$ at $x$ if for all $i \in N$, $p \cdot x_{i}^{\prime} \geq p \cdot x_{i}$ for all $x_{i}^{\prime} \in V\left(x_{i}, u_{i}\right)$. The set of supporting prices for $u$ at $x$ is denoted by $\Pi(x, u)$.

According to Definition 3, the supporting prices for $u$ at $x$ are equally important to allocation rules in $\mathcal{F}$. This is, however, not completely satisfactory since a supporting price in $\Pi(x, u)$ may be more economically important than others in relation to the efficient allocation rule $F$ but less important than others in relation to another efficient allocation rule. Motivated by this, we develop below a definition of supporting prices that possibly also depends on the efficient allocation rule considered. Our development starts with the following definition.

Definition 4. Let $u \in U_{N}, x \in Q^{n}$ and $F \in \mathcal{F}$ be given. A supporting price $p$ for $u$ at $x$ is said to be a supporting price for $u$ and $F$ at $x$ if there exists $u^{\prime} \in U_{N}$ 
such that (i) $x$ is an $F$-allocation at $u^{\prime}$ as well as at $u$, and (ii) $p$ is the unique supporting price for $u^{\prime}$ at $x$. The set of supporting prices for $u$ and $F$ at $x$ is denoted by $\Pi^{F}(x, u)$.

Within a framework that includes linear utility functions, requirement (ii) of Definition 4 is satisfied. More difficult, however, is satisfying requirement (i). In order to deal with the non-existence of supporting prices that meets both requirements, our subsequent approach is to consider the definition of supporting prices in Saijo et al. (1999) provided that no supporting price for $u$ at $x$ passes the test of Definition 4 .

Definition 5. Let $u \in U_{N}, x \in Q^{n}$ and $F \in \mathcal{F}$ be given. Define the set of supporting prices for $u$ and possibly for $F$ at $x$ to be $\pi^{F}(x, u)=\Pi^{F}(x, u)$ if $\Pi^{F}(x, u)$ is non-empty, or else to be $\pi^{F}(x, u)=\Pi(x, u)$.

\subsection{Semi-responsible agents}

A classic postulate of implementation theory in economic environments is that agents only care about quantity(ies) obtained from the mechanism. It is not unrealistic, however, to also assume that agents involved in a mechanism may have intrinsic preferences for responsibility. There are several ways to model intrinsic preferences for responsibility within a $p-q$ mechanism. In this paper, we adopt the following notion of responsible actions.

Definition 6. Let $u \in U_{N}, F \in \mathcal{F}$ and $i \in N$ be given. A price vector $p^{i} \in P$ and a quantity $x_{i}^{i} \in Q$ (announced by $i$ ) are said to be responsible for $(u, F)$ if there exists a profile of quantities $x_{-i} \in Q^{n-1}$ such that (i) $\left(x_{i}^{i}, x_{-i}\right)$ is an $F$-allocation at $u$ and (ii) $p^{i}$ is a supporting price for $u$ and possibly for $F$ at $\left(x_{i}^{i}, x_{-i}\right)$. We say that the (announced) pair $\left(p^{i}, x_{i}^{i}\right)$ is responsible for $(u, F)$ if $p^{i}$ and $x_{i}^{i}$ are responsible for $(u, F)$.

It follows that the price vector announcement and the quantity announcement are both relevant to our notion of responsibility. As an example, let us consider the Walrasian rule, denoted by $W$, that assigns to each profile $u$ the corresponding set of Walrasian equilibrium allocations. ${ }^{8}$ A price-quantity pair $\left(p^{i}, x_{i}^{i}\right)$ is responsible for $(u, W)$ if there is a profile of quantities $x_{-i} \in Q^{n-1}$ such that the allocation $\left(x_{i}^{i}, x_{-i}\right)$ is a Walrasian equilibrium allocation for $u$ and the price vector $p^{i}$ is the Walrasian equilibrium price vector associated with that allocation.

Although our notion of responsibility is not the weakest notion one could think of, casual introspection indicates that it is plausible for an agent to act responsibly for the benefit of society at large when a lie does not lead to any better outcome, and hence in modeling intrinsic preferences for responsibility we take the view of Dutta and Sen (2012). Intuitively, for any given pair $(u, F)$, an agent has an intrinsic preference for responsibility if she prefers to announce a responsible price-quantity pair for $(u, F)$ when a lie does not better serve her material interests, given the

8. For a formal definition see section 4 . 
announcements made by other agents. Operationally, for each pair $(u, F)$, we need to extend every agent $i$ 's utility function $u_{i}$ to an ordering over the strategy space $M$, because the agent $i$ 's preference between being responsible and being not is contingent upon announcements made by other agents as well as the quantity(ies) obtained from them. By following standard conventions of preference orderings, let $R_{i}^{\gamma}[u, F]$ denote agent $i$ 's ordering (extension of $u_{i}$ ) over the strategy space $M$ at the pair $(u, F)$ for a given $p-q$ mechanism $\gamma$. The corresponding strict and indifference relations are denoted by $P_{i}^{\gamma}[u, F]$ and $I_{i}^{\gamma}[u, F]$, respectively.

Definition 7. Given a price-quantity mechanism $\gamma=(M, g)$, agent $i \in N$ is said to be semi-responsible if for every $(u, F) \in U_{N} \times \mathcal{F}$ there exists an ordering $R_{i}^{\gamma}[u, F]$ over $M$ such that for every $m_{-i} \in M_{-i}$ and every $m_{i}, m_{i}^{\prime} \in M_{i}$ it holds that:

(i) If $m_{i}$ is responsible for $(u, F)$ and $m_{i}^{\prime}$ is not responsible for $(u, F)$ and if $u_{i}\left(g_{i}\left(m_{i}, m_{-i}\right)\right) \geq u_{i}\left(g_{i}\left(m_{i}^{\prime}, m_{-i}\right)\right)$, then $\left(m_{i}, m_{-i}\right) P_{i}^{\gamma}[u, F]\left(m_{i}^{\prime}, m_{-i}\right)$.

(ii) In all other cases,

$$
u_{i}\left(g_{i}\left(m_{i}, m_{-i}\right)\right) \geq u_{i}\left(g_{i}\left(m_{i}^{\prime}, m_{-i}\right)\right) \text { if and only if }\left(m_{i}, m_{-i}\right) R_{i}^{\gamma}[u, F]\left(m_{i}^{\prime}, m_{-i}\right) .
$$

Intrinsic preferences for responsibility of agent $i$ are captured by the first part of Definition 7 , in that for every pair $(u, F)$ agent $i$ strictly prefers the profile $\left(m_{i}, m_{-i}\right)$ to $\left(m_{i}^{\prime}, m_{-i}\right)$ according to $R_{i}^{\gamma}[u, F]$ provided that agent $i$ 's true utility at the quantity $g_{i}\left(m_{i}^{\prime}, m_{-i}\right)$ is not larger than the true utility at $g_{i}\left(m_{i}, m_{-i}\right)$ and provided that her price-quantity announcement is responsible for $(u, F)$ in $\left(m_{i}, m_{-i}\right)$ and not responsible for $(u, F)$ in $\left(m_{i}^{\prime}, m_{-i}\right)$.

If agent $i$ is not semi-responsible, this agent cares about the levels of utility associated with different quantities and nothing else. Then, agent $i$ 's ordering over $M$ is just the transposition into space $M$ of agent $i$ 's relative ranking of quantities. Formally:

Definition 8. Given a price-quantity mechanism $\gamma=(M, g)$, agent $i \in N$ is said to be not semi-responsible if for every $(u, F) \in U_{N} \times \mathcal{F}$ there exists an ordering $R_{i}^{\gamma}[u, F]$ over $M$ such that for every $m, m^{\prime} \in M$ it holds that

$$
m R_{i}^{\gamma}[u, F] m^{\prime} \text { if and only if } u_{i}\left(g_{i}(m)\right) \geq u_{i}\left(g_{i}\left(m^{\prime}\right)\right) .
$$

\subsection{Mechanism designer's problem}

In this subsection, we define and discuss the mechanism designer's Nash implementation problem with semi-responsible agents as well as the notion of a natural $p-q$ mechanism. In formalizing the mechanism designer's problem, we first introduce our informational assumptions and discuss their implications for our analysis.

Assumption 1. There is at least one semi-responsible agent in $N$. 
Further, we assume that there is complete information among the agents. Then, the set of agents, the endowment profile, the objective of the mechanism designer represented by $F$, the true utility functions of agents, their rationality as well as the identity of semi-responsible agents are informally understood to be common knowledge among the agents.

In our framework, these informational assumptions mean the mechanism designer knows the set of allocations, as well as the fact that there are semi-responsible agents among the agents, but knows neither the true utility functions of agents nor the identity of the semi-responsible agent (or their identities). Indeed, the mechanism designer cannot exclude any subset of agents from being semi-responsible purely on the basis of Assumption 1. Therefore, from the point of view of the mechanism designer:

- A possible state of the world is described by three parameters: an allowable set of semi-responsible agents, an allocation rule $F$ as well as a profile of utility functions. We denote by $H$ a typical set of semi-responsible agents in $N$ and by $\mathcal{H}$ the class of conceivable sets of semi-responsible agents.

- For any $p-q$ mechanism $\gamma$, each possible state $(u, F, H)$ is associated with a profile of agents' orderings $R^{\gamma}[u, F, H] \equiv\left(R_{1}^{\gamma}[u, F], \ldots, R_{n}^{\gamma}[u, F]\right)$, where $R_{i}^{\gamma}[u, F]$ is the agent $i$ 's ordering over $M$ as formulated in Definition 7 if agent $i$ is in $H$, whereas it is the agent $i$ 's ordering over $M$ as formulated in Definition 8 if agent $i$ is not in $H$.

- A $p$ - $q$ mechanism $\gamma$ and a state $(u, F, H)$ induce a strategic game $\left(\gamma, R^{\gamma}[u, F, H]\right)$.

- A (pure strategy) Nash equilibrium of the strategic game $\left(\gamma, R^{\gamma}[u, F, H]\right)$ is a message profile $m$ such that for each agent $i \in N, m R_{i}^{\gamma}[u, F]\left(m_{i}^{\prime}, m_{-i}\right)$ for every $m_{i}^{\prime} \in M_{i}$. We denote by $N E\left(\gamma, R^{\gamma}[u, F, H]\right)$ the set of Nash equilibrium message profiles of $\left(\gamma, R^{\gamma}[u, F, H]\right)$, whereas $N A\left(\gamma, R^{\gamma}[u, F, H]\right)$ is its corresponding set of Nash equilibrium allocations.

In this paper, we also ask the mechanism designer to design a $p-q$ mechanism satisfying the following properties. Firstly, the allocation of the mechanism is to be individually feasible. That is,

Individual feasibility For all $m \in M, g(m) \in \mathbb{R}_{+}^{n \ell}$.

Secondly, the total supply of commodity $l$ to agents cannot exceed its aggregate endowment $\Omega_{l}$. That is,

Weak balancedness For all $l \in L$ and $m \in M, g_{1, l}(m)+\ldots+g_{n, l}(m) \leq \Omega_{l}$.

Thirdly, for any strategy profile of other agents $m_{-i}$, each agent $i$ needs to have a best response strategy. That is, 
Best response property (Jackson, Palfrey and Srivastava, 1994) For all $i \in N$, $u \in U_{N}$ and $m_{-i} \in M_{-i}$, there exists $m_{i} \in M_{i}$ such that $u_{i}\left(g_{i}\left(m_{i}, m_{-i}\right)\right) \geq$ $u_{i}\left(g_{i}\left(m_{i}^{\prime}, m_{-i}\right)\right)$ for every $m_{i}^{\prime} \in M_{i}$.

Lastly, in equilibrium, each agent needs to receive the quantity demanded. That is,

Forthrightness (Saijo, Tatamitani and Yamato, 1996, 1999) For all $(u, F, H), x \in$ $F(u)$ and $p \in \pi^{F}(x, u)$, the price-quantity strategy profile $\left(p, x_{i}\right)_{i \in N}$ is in $N E\left(\gamma, R^{\gamma}[u, F, H]\right)$ and $g\left(\left(p, x_{i}\right)_{i \in N}\right)=x .^{9}$

We call any $p-q$ mechanism that satisfies the above four properties a natural $p-q$ mechanism (with free disposal) following the literature on $p-q$ mechanisms.

The following definition represents our formulation of the mechanism designer's Nash implementation problem with semi-responsible agents when only natural $p-q$ mechanisms can be devised.

Definition 9. Let Assumption 1 and $F \in \mathcal{F}$ be given. A natural $p-q$ mechanism $\gamma$ semi-responsibly Nash implements $F$ if $F(u)=N A\left(\gamma, R^{\gamma}[u, F, H]\right)$ for all $u \in U_{N}$ and $H \in \mathcal{H}$. If such a mechanism exists, $F$ is said to be semi-responsibly Nash implementable by natural $p-q$ mechanisms.

The objective of the mechanism designer is thus to design a natural $p-q$ mechanism whose Nash equilibrium allocations, for each environment $u$ as well as each conceivable set of semi-responsible agents $H$, coincide with $F(u)$. Interestingly, there is no distinction between the above formulation and the standard Nash implementation problem as long as Assumption 1 is violated.

\section{Results}

In this section, we first propose a necessary and sufficient condition for Nash implementation by a natural $p-q$ mechanism when there exists at least one semi-responsible agent. Then, we present our characterization result. Finally, we show that the Walrasian rule satisfies our condition and that our condition imposes non-trivial restrictions on the class of efficient allocation rules.

\subsection{A characterization result}

There are two key considerations underpinning our condition. These are presented from the viewpoint of necessity. To this end, suppose an efficient allocation rule $F$ is semi-responsibly Nash implementable by a natural $p-q$ mechanism $\gamma \equiv(M, g)$.

The first important difficulty for the mechanism designer is to decide what allocation to assign to a strategy profile when agents announce the same price vector, that

9. See also Dutta, Sen and Vohra (1995). Similar properties are used also in general environments; see, e.g., Tatamitani (2001) and Lombardi and Yoshihara (2013a). 
is, $m_{i}=\left(p, x_{i}\right)$ for every agent $i$. Following Saijo, Tatamitani and Yamato (1996; $1999)$, a clue to this issue is given by the allocation $\left(\Omega-\sum_{j \neq i} x_{j}, x_{-i}\right)$, where quantities of agents other than agent $i$ are fixed at their announced levels and agent $i$ 's quantity is the remainder, $x_{i}^{\Omega} \equiv \Omega-\sum_{j \neq i} x_{j}$. If the mechanism designer can find an environment $u$ for which $\left(x_{i}^{\Omega}, x_{-i}\right)$ is an $F$-allocation at $u$ and the price vector $p$ is a supporting price for $u$ and possibly for $F$ at $\left(x_{i}^{\Omega}, x_{-i}\right)$, the designer infers from this that agent $i$ is a potential deviator for $F$ at $\left(x_{1}, \ldots, x_{n}\right)$. The reason is agent $i$ should have announced the quantity $x_{i}^{\Omega}$ rather than $x_{i}$. On the other hand, if no such environments exist, the mechanism designer can only conclude that agent $i$ and the other agents had demanded the wrong quantities. Therefore, agent $i$ cannot be identified as a potential deviator. Note there may be many potential deviators. For instance, if the announced quantity profile $\left(x_{i}^{\Omega}, x_{-i}\right)$ for each $i$ were respectively an $F$-allocation at $u$ and the price vector $p$ were a supporting price for $u$ and possibly for $F$ at $\left(x_{i}^{\Omega}, x_{-i}\right)$ for each $i$ respectively, then every agent would be a potential deviator.

Hence, our condition requires the existence of a feasible and weakly balanced punishment allocation $\mathbf{z}(p, x) \in A$ when all agents are potential deviators. Agent $i$ 's punishment quantity $\mathbf{z}_{i}(p, x)$ is selected as follows. Since agent $i$ is a potential deviator, there exists $u$ such that $\left(x_{i}^{\Omega}, x_{-i}\right) \in F(u)$ and $p \in \pi^{F}\left(\left(x_{i}^{\Omega}, x_{-i}\right), u\right)$. Moreover, forthrightness ensures that the strategy profile made up of $m_{i}^{\prime}=\left(p, x_{i}^{\Omega}\right)$ and $m_{-i} \equiv\left(m_{1}, \ldots, m_{i-1}, m_{i+1}, \ldots, m_{n}\right)$ is a Nash equilibrium profile at $u$ and $g\left(m_{i}^{\prime}, m_{-i}\right)$ is equal to $\left(x_{i}^{\Omega}, x_{-i}\right)$. Hence, the attainable set $g_{i}\left(M_{i}, m_{-i}\right)$ of agent $i$ is contained in the weak lower contour set of $u_{i}$ at $x_{i}^{\Omega}$. Since there can be more than one environment having the features of $u$ and, moreover, the mechanism designer cannot exclude any of such environments from being the true one, the preceding line of reasoning leads to the conclusion that the attainable set $g_{i}\left(M_{i}, m_{-i}\right)$ is contained in the intersection of agent $i$ 's weak lower contour sets of all such environments at $x_{i}^{\Omega}$. Agent $i$ 's punishment quantity can be set equal to $g_{i}\left(m_{i}, m_{-i}\right)$.

Formally, let $p \in P$ and $x_{i} \in Q$ for every agent $i$ be given. Let $x \equiv\left(x_{1}, \ldots, x_{n}\right)$ denote the announced quantity profile and let $x_{i}^{\Omega} \equiv \Omega-\sum_{j \neq i} x_{j}$ denote agent $i$ 's remainder. The set of environments for which agent $i$ is a potential deviator is defined by $F^{-1}\left(\left(x_{i}^{\Omega}, x_{-i}\right), p\right) \equiv\left\{u \in U_{N} \mid\left(x_{i}^{\Omega}, x_{-i}\right) \in F(u)\right.$ and $\left.p \in \pi^{F}\left(\left(x_{i}^{\Omega}, x_{-i}\right), u\right)\right\}$. For any of such environments, the weak lower contour set of $u_{i}$ at $x_{i}^{\Omega}$ is defined by $L\left(x_{i}^{\Omega}, u_{i}\right) \equiv\left\{x_{i}^{\prime} \in Q \mid u_{i}\left(x_{i}^{\Omega}\right) \geq u_{i}\left(x_{i}^{\prime}\right)\right\}$, whereas their intersection is defined by

$$
\Lambda_{i}^{F}\left(\left(x_{i}^{\Omega}, x_{-i}\right), p\right) \equiv \bigcap_{u \in F^{-1}\left(\left(x_{i}^{\Omega}, x_{-i}\right), p\right)} L\left(x_{i}^{\Omega}, u_{i}\right) .
$$

The set of potential deviators for price-quantity Nash implementation is defined by $I^{F}(p, x) \equiv\left\{i \in N \mid F^{-1}\left(\left(x_{i}^{\Omega}, x_{-i}\right), p\right) \neq \varnothing\right\}$. Hence:

(i) If $I^{F}(p, x)=N$, then there exists $\mathbf{z}(p, x) \in A$, with $\mathbf{z}(p, x)=x$ if $\sum_{i \in N} x_{i}=\Omega$, such that $\mathbf{z}_{i}(p, x) \in \Lambda_{i}^{F}\left(\left(x_{i}^{\Omega}, x_{-i}\right), p\right)$ for every $i \in N$.

As already noted, a semi-responsible agent $i$ 's preference between being responsible and being not responsible depends on strategies played by other agents as well as the 
quantity(ies) obtained from them. The second important difficulty for the mechanism designer is to decide what quantity to assign to agent $i$ when the latter plays a responsible strategy while the other agents' strategy choices are kept fixed. Hence, we require the existence of suitable quantities to assign to agent $i$ when agent $i$ plays a responsible strategy $m_{i}$ and every other agent $j$ plays $m_{j}=\left(p, x_{j}\right)$.

Since the existence of a suitable quantity for agent $i$ is ensured by condition (i) when all agents are potential deviators at $\left(m_{i}, m_{-i}\right)$, the following condition (ii) encompasses cases where, instead, not all agents are potential deviators at that profile. ${ }^{10}$ That is, for any given $p \in P$ and $x \in Q^{n}$ :

(ii) If $1 \leq \# I^{F}(p, x) \leq n-1$, then there exists $\overline{\mathbf{z}}(p, x) \in A$ such that for each $i \in N, \overline{\mathbf{z}}_{i}(p, x) \in \Lambda_{i}^{F}\left(\left(x_{i}^{\Omega}, x_{-i}\right), p\right)$ if $i \in I^{F}(p, x)$ and the announcement $\left(p, x_{i}\right)$ is responsible for some allowable $\left(u^{*}, F\right)$, or else $\overline{\mathbf{z}}_{i}(p, x)=0$.

Note the above condition assigns the worst possible quantity to agents who are not potential deviators at $(p, x)$ or play strategies that are not responsible for the entire economic domain $U_{N}$. Furthermore, condition (ii) is a necessary condition for implementation. To see this, observe that we can repeat the same arguments used above to obtain that $g_{i}\left(M_{i}, m_{-i}\right) \subseteq \Lambda_{i}^{F}\left(\left(x_{i}^{\Omega}, x_{-i}\right), p\right)$ for every agent $i \in I^{F}(p, x)$. We can then define agent $i$ 's quantity $\overline{\mathbf{z}}_{i}(p, x)$ as follows: If $i \in I^{F}(p, x)$ and $m_{i}=\left(p, x_{i}\right)$ is responsible for some $\left(u^{*}, F\right)$, then $\overline{\mathbf{z}}_{i}(p, x)=g_{i}(m)$ or else, $\overline{\mathbf{z}}_{i}(p, x)=0$. The verification of condition (ii) follows immediately from that definition.

Beside cases in which agents state the same prices, the mechanism designer needs to support responsibility in cases where agent $i$ reports prices that do not match with those reported by other agents. In cases like this, the existence of a suitable quantity for agent $i$ is ensured by condition (iii) below. That is, for any given $p \in P$ and $x \in Q^{n}:$

(iii) For any $i \in N$, there exists a map $z_{i}\left(\cdot ;\left(p, x_{-i}\right)\right): P \times Q \rightarrow Q$ such that for every $\left(p^{\prime}, x_{i}^{\prime}\right)$,

(a) If $\left(p^{\prime}, x_{i}^{\prime}\right)$ is responsible for some allowable $\left(u^{*}, F\right)$ and $i \in I^{F}\left(p,\left(x_{i}^{\prime}, x_{-i}\right)\right)$, then $z_{i}\left(\left(p^{\prime}, x_{i}^{\prime}\right) ;\left(p, x_{-i}\right)\right) \in \Lambda_{i}^{F}\left(\left(x_{i}^{\Omega}, x_{-i}\right), p\right)$, with $z_{i}\left(\left(p^{\prime}, x_{i}^{\prime}\right) ;\left(p, x_{-i}\right)\right)=\mathbf{z}_{i}(p, x)$ if $p^{\prime}=p$ and $I^{F}\left(p,\left(x_{i}^{\prime}, x_{-i}\right)\right)=N$, and with $z_{i}\left(\left(p^{\prime}, x_{i}^{\prime}\right) ;\left(p, x_{-i}\right)\right)=\overline{\mathbf{z}}_{i}\left(p,\left(x_{i}^{\prime}, x_{-i}\right)\right)$ if $p^{\prime}=p$ and $1 \leq \# I^{F}\left(p,\left(x_{i}^{\prime}, x_{-i}\right)\right) \leq n-1$.

(b) Otherwise, $z_{i}\left(\left(p^{\prime}, x_{i}^{\prime}\right) ;\left(p, x_{-i}\right)\right)=0$.

Note that when the premises of condition (i) or (ii) are also met, the quantity selected by the map $z_{i}\left(\cdot ;\left(p, x_{-i}\right)\right)$ coincides with the quantity assigned to agent $i$ by, respectively, $\mathbf{z}_{i}(p, x)$ and $\overline{\mathbf{z}}_{i}(p, x)$. Moreover, in the same spirit of condition (ii), condition (iii) assigns the worst possible quantity to agent $i$ when she plays a strategy that is not responsible for the entire domain. However, unlike the first two conditions, condition (iii) does not require the existence of an allocation.

10. For a set $S$, we write $\# S$ to denote the number of elements in $S$. 
In light of our discussion on the existence of the punishment allocation $\mathbf{z}(p, x)$ of condition (i), it can be seen that $g_{i}\left(\left(p^{\prime}, x_{i}^{\prime}\right), m_{-i}\right) \in \Lambda_{i}^{F}\left(\left(x_{i}^{\Omega}, x_{-i}\right), p\right)$ if agent $i$ is a potential deviator at $\left(p,\left(x_{i}^{\prime}, x_{-i}\right)\right)$ and, moreover, $\left(p^{\prime}, x_{i}^{\prime}\right)$ is a responsible strategy. We can then define $z_{i}\left(\left(p^{\prime}, x_{i}^{\prime}\right) ;\left(p, x_{-i}\right)\right)$ by setting it equal to $g_{i}\left(\left(p^{\prime}, x_{i}^{\prime}\right), m_{-i}\right)$ if $\left(p^{\prime}, x_{i}^{\prime}\right)$ is responsible for some allowable $\left(u^{*}, F\right)$ and $i \in I^{F}\left(p,\left(x_{i}^{\prime}, x_{-i}\right)\right)$, or else equal to the worst possible quantity. Again, the verification of condition (iii) follows immediately from that definition. This definition also specifies the property of $z_{i}\left(\cdot ;\left(p, x_{-i}\right)\right)$ as satisfying $z_{i}\left(\left(p^{\prime}, x_{i}^{\prime}\right) ;\left(p, x_{-i}\right)\right) \in \Lambda_{i}^{F}\left(\left(x_{i}^{\Omega}, x_{-i}\right), p\right)$ for the case that $\left(p^{\prime}, x_{i}^{\prime}\right)$ is responsible for some allowable $\left(u^{*}, F\right), i \in I^{F}\left(p,\left(x_{i}^{\prime}, x_{-i}\right)\right)$, and $p^{\prime} \neq p$.

The condition below states that if the punishment allocation $\mathbf{z}(p, x)$ of condition (i) is an equilibrium allocation of the strategic game $\left(\gamma, R^{\gamma}\left[u^{*}, F, H\right]\right)$, then it must be an $F$-allocation at $u^{*}$. Expressed differently, if the punishment allocation $\mathbf{z}(p, x)$ of condition (i) is not an $F$-allocation at $u^{*}$ and moreover, no agent $j$ has a material incentive to unilaterally deviate from $\mathbf{z}_{j}(p, x)$, then at least one semi-responsible agent in $H$ is acting not responsibly and at least one of these agents can switch to a strategy that is responsible for $\left(u^{*}, F\right)$ and gives him/her no material loss. That is, for any given $p \in P$ and $x \in Q^{n}$ :

(iv) For any $u^{*} \in U_{N}$ and $H \in \mathcal{H}$, if $I^{F}(p, x)=N, \Lambda_{j}^{F}\left(\left(x_{j}^{\Omega}, x_{-j}\right), p\right) \subseteq L\left(\mathbf{z}_{j}(p, x), u_{j}^{*}\right)$ for every $j \in N$, and $\mathbf{z}(p, x) \notin F\left(u^{*}\right)$, then there is an agent $i \in H$ such that (a) $\left(p, x_{i}\right)$ is not responsible for $\left(u^{*}, F\right)$; and (b) there exists $\left(p^{\prime}, x_{i}^{\prime}\right)$ that is responsible for $\left(u^{*}, F\right)$ such that $u_{i}^{*}\left(z_{i}\left(\left(p^{\prime}, x_{i}^{\prime}\right) ;\left(p, x_{-i}\right)\right)\right)=u_{i}^{*}\left(\mathbf{z}_{i}(p, x)\right)$.

We have already seen that if $m_{j}=\left(p, x_{j}\right)$ for every agent $j$, then $g_{j}\left(M_{j}, m_{-j}\right) \subseteq$ $\Lambda_{j}^{F}\left(\left(x_{j}^{\Omega}, x_{-j}\right), p\right)$ and $\mathbf{z}(p, x)=g(m)$ when all agents are potential deviators at $m$. To verify condition (iv), suppose $u^{*}$ is a true environment, $\mathbf{z}(p, x)$ is not an $F$ allocation at $u^{*}$ and no quantity in $\Lambda_{j}^{F}\left(\left(x_{j}^{\Omega}, x_{-j}\right), p\right)$ is strongly preferred by agent $j$ to $\mathbf{z}_{j}(p, x)$. Then, no agent has a material incentive to unilaterally deviate from $g(m)$. By implementability, we then have that for at least one agent $i \in H$, the strategy $\left(p, x_{i}\right)$ is not responsible for $\left(u^{*}, F\right)$ and the mechanism is such that it makes responsible reporting non-costly for this agent, in the sense that $u_{i}^{*}\left(g_{i}\left(\left(p^{\prime}, x_{i}^{\prime}\right), m_{-i}\right)\right)=$ $u_{i}^{*}\left(\mathbf{z}_{i}(p, x)\right)$ for a strategy $\left(p^{\prime}, x_{i}^{\prime}\right)$ responsible for $\left(u^{*}, F\right)$. Observe that agent $i$ is a potential deviator at $\left(p,\left(x_{i}^{\prime}, x_{-i}\right)\right)$ and so $g_{i}\left(\left(p^{\prime}, x_{i}^{\prime}\right), m_{-i}\right)=z_{i}\left(\left(p^{\prime}, x_{i}^{\prime}\right) ;\left(p, x_{-i}\right)\right)$ by condition (iii).

In summary, if the efficient allocation rule $F$ is semi-responsibly Nash implementable by a natural $p-q$ mechanism, then the following condition, which is a variant of the monotonicity condition and of the punishment condition due to Saijo, Tatamitani and Yamato $(1996,1999)$, must be satisfied:

Condition M-PQ: Let $F \in \mathcal{F}$ be given. Let $p \in P$ and $x \in Q^{n}$ be given. Let $x_{i}^{\Omega} \equiv \Omega-\sum_{j \neq i} x_{j}$ denote agent $i$ 's remainder. Then, (i), (ii), (iii) and (iv) are satisfied.

Condition M-PQ is not only necessary but is also sufficient. 
Theorem 1. Let $n \geq 3$. Let Assumption 1 and $F \in \mathcal{F}$ be given. $F$ is semiresponsibly Nash implementable by a natural $p-q$ mechanism if and only if it satisfies condition M-PQ.

Proof. See Appendix.

In the sufficiency part of Theorem 1, we constructed a mechanism that resembles that of Saijo et al. $(1996,1999)$. However, unlike theirs, ours does not rely on any sort of "tail-chasing" construction. ${ }^{11}$ We are able to do so because in our framework there are semi-responsible agents as well as the allowance of free-disposal.

Remark 1. The devised implementing $p-q$ mechanism is not balanced since out of equilibrium the total demand is not necessarily equal to the total supply. Lombardi and Yoshihara (2013b) successfully constructed a balanced $p-q$ mechanism, but this construction relies on the so-called "modulo game". As is well known, in such games, mixed strategy Nash equilibria do exist and they are ruled out only by assumption. To avoid this difficulty, the so-called "integer game" can be used to construct a balanced $p-q$ mechanism. Horeover, since each agent chooses a positive integer in addition to a price vector $p$ and a quantity $q$ as her strategy choice, that mechanism would not resemble any real-life mechanism. Sjöström (1996) successfully devised a market mechanism that does not use any sort of "integer game" or "modulo game", but his mechanism does not satisfy neither the best response property nor balancedness. Dutta and Sen (2012) succeeded in constructing a feasible and bounded (in the sense of Jackson, 1992) direct mechanism using neither a "modulo game" nor an "integer game", but their mechanism is neither balanced (out of equilibrium) nor a market mechanism (see Dutta and Sen, 2012; Remark 4). This latter comment also extends to Kartik et al. (2014)'s mechanism. Therefore, as already noted by Saijo et al. (1996; p. 971), this suggests that the property of balancedness may not be compatible with a $p-q$ mechanism that is not subject to the critiques that are leveled against the mechanisms used for Nash implementation. However, a definitive answer to this compatibility issue is still unknown.

\subsection{Natural implementation of the Walrasian rule}

Let us turn now to a brief discussion of the implications of Theorem 1. As an example of efficient allocation rules in $\mathcal{F}$ satisfying our condition, we focus on the Walrasian rule which we define next.

Walrasian rule, $W$ : For each $u \in U, W(u) \equiv\{x \in A \mid$ there exists $p \in P$ such that for each $i \in N$, it holds $p \cdot x_{i}=p \cdot \omega_{i}$ and for each $y_{i} \in \mathbb{R}_{+}^{\ell}, u_{i}\left(y_{i}\right)>u_{i}\left(x_{i}\right)$ implies $p \cdot y_{i}>p \cdot \omega_{i}$, where $U$ is the class of all profiles of utility functions that are continuous, quasi-concave and strongly monotonic on $\mathbb{R}_{+}^{\ell}$.

11. See Maskin (1999). For the criticism against such construction, see Jackson (1992). 
We show below that the Walrasian rule satisfies condition M-PQ. We begin with an outline of its proof for the case where the profile $x \in Q^{n}$ is totally balanced and all agents are potential deviators for $W$ at $(p, x)$. In that case we can define the punishment allocation of condition (i) by $\mathbf{z}(p, x)=x .^{12}$ Further, we take any $u \in W^{-1}(p, x)$ and then show (on the basis that $W$ is defined on the 'unrestricted' domain $U$ ) that the vector $p$ is a supporting price for $u$ and for $W$. This leads to the conclusion that for any agent $i$, the set $\Lambda_{i}^{W}(x, p)$ is agent $i$ 's constrained budget set.

Next, we fix an arbitrary environment $u^{*}$ where, for any agent $i$, the quantity $x_{i}$ maximizes agent $i$ 's utility $u_{i}^{*}$ over $\Lambda_{i}^{W}(x, p)$. For the premises of condition (iv) to be met, we also assume $x$ is not a Walrasian allocation at $u^{*}$. This means that there exists an agent $k$ for whom the quantity $x_{k}$ is not a utility maximizing quantity (in terms of $\left.u_{k}^{*}\right)$ over the budget set for the given pair $\left(p, \omega_{k}\right)$. We then also observe that the price vector $p$ cannot be an equilibrium price for $u^{*}$ - simply because any solution to agent $k$ 's maximization problem over the (unconstrained) budget set given by the pair $\left(p, \omega_{k}\right)$ occurs outside the set $Q$. This allows us to establish that for every agent $i$, the price-quantity pair $\left(p, x_{i}\right)$ cannot be a responsible pair for $\left(u^{*}, W\right)$, and so establish part (a) of condition (iv).

To prove part (b) of condition (iv), there are two things to prove: first, that the map $z_{i}\left(\cdot ;\left(p, x_{-i}\right)\right)$ meets the requirements of condition (iii) and, second, that $u_{i}^{*}\left(\mathbf{z}_{i}(p, x)\right)=u_{i}^{*}\left(z_{i}\left(\left(p^{*}, x_{i}^{*}\right) ;\left(p, x_{-i}\right)\right)\right)$ for a pair $\left(p^{*}, x_{i}^{*}\right)$ that is responsible for $\left(u^{*}, W\right)$. In the definition of $z_{i}\left(\cdot ;\left(p, x_{-i}\right)\right)$, we use the important insight that no responsible price-quantity pairs for $\left(u^{*}, W\right)$ can encompass the price vector $p$. In fact, the key requirement behind its definition is that for agent $i$ 's responsible pricequantity pairs $\left(p^{\prime}, x_{i}^{\prime}\right)$ for $\left(u^{*}, W\right)$ the map should be unresponsive, in the sense that $z_{i}\left(\left(p^{\prime}, x_{i}^{\prime}\right) ;\left(p, x_{-i}\right)\right)=\mathbf{z}_{i}(p, x)$. In turn, the completion of the proof of part (b) follows directly from that property of the map $z_{i}\left(\cdot ;\left(p, x_{-i}\right)\right)$. We will now summarize this as follows.

Theorem 2. Let $n \geq 3$. Let Assumption 1 be given. Then, the Walrasian rule satisfies condition M-PQ.

Proof. See Appendix.

Remark 2. It bears mentioning again here that Theorem 2 is built on a definition of natural $p-q$ mechanisms that differs from that in Saijo et al. (1999). This is so because the requirement of forthrightness is based upon a notion of supporting prices that depends on the efficient allocation rule considered. As already noted, this choice is dictated by the consideration that supporting prices are not equally important to every efficient allocation rule. This is particularly true for the Walrasian rule. Indeed, Theorem 2 fails to hold if we focus on the set of supporting prices of Definition 3 and we base the requirement of forthrightness as well as the notion of responsible reports upon this set. This can be shown by means of the following example.

12. Where $x$ was not a totally balanced allocation, we could define the punishment allocation of condition (i) by $\mathbf{z}(p, x)=0$. In that case condition (iv) is satisfied (vacuously) where $I^{W}(p, x)=N$. 
As in Saijo et al. (1999), let us base the condition of forthrightness and the set of environments for which agent $i$ is a potential deviator, that is, the definition of $F^{-1}\left(\left(x_{i}^{\Omega}, x_{-i}\right), p\right)$ (see p. 10), on the set of supporting prices of Definition 3. Furthermore, let us use this definition of supporting prices as the basis of responsible strategies, that is, of Definition 6. With obvious adaptations of the proof of Theorem 1, one can see that (the new) condition M-PQ is a necessary condition for Nash implementation with semi-responsible agents by a Saijo et al. (1999)'s natural $p-q$ mechanism. With these preliminaries in place, we are now in a position to show the example we mentioned in the preceding paragraph.

Example 1. For convenience, consider a setting with three agents and three commodities. The initial endowment of commodities is $\omega_{i}=(1,1,1)$ for every agent $i$. Consider the following quantities:

$$
\begin{array}{lll}
\text { for agent } 1: & x_{1}=(3,0,1), \\
\text { for agent } 2: & x_{2}=(0,3,0), \\
\text { for agent } 3: & x_{3}=(0,0,2) .
\end{array}
$$

Note that the point $x=\left(x_{1}, x_{2}, x_{3}\right)$ is a boundary (balanced) allocation.

We can find a preference profile $u \in U$ such that $x$ is a Walrasian allocation at $u$ with the equilibrium price $p^{W}=(1 / 6,1 / 3,1 / 2)$ and such that agent $i$ 's preference $u_{i}$ is not differentiable at the quantity $x_{i}$. This implies that the price vector $p=((1 / 6)+(5 / 3) \varepsilon,(1 / 3)-(2 / 3) \varepsilon,(1 / 2)-\varepsilon)$ is an element of $\Pi(x, u)$ where $\varepsilon>0$ is arbitrarily small. Observe that agent $i$ 's budget constraint is violated at the price vector $p$, that is, $p \cdot x_{i} \neq p \cdot \omega_{i}$, and so this $p$ is not a supporting price for $u$ and $W$ at $x$; that is, $p \notin \Pi^{W}(x, u)$. Also, note that for any agent $i,\left(p, x_{i}\right)$ is a responsible report for $u$ and $W$ given that $x \in W(u)$ and $p \in \Pi(x, u)$.

In addition, consider the following quantities:

$$
\begin{array}{lll}
\text { for agent } 1: & a_{1}=x_{1}, \quad b_{1}=(2,0,0) \quad \text { and } \quad c_{1}=(3,0,1 / 2), \\
\text { for agent } 2: & a_{2}=(0,2,0), \quad b_{2}=x_{2} \quad \text { and } \quad c_{2}=(0,3,1 / 2), \\
\text { for agent } 3: & a_{3}=(0,1,2), \quad b_{3}=(1,0,3) \quad \text { and } \quad c_{3}=x_{3} .
\end{array}
$$

Note that the points $a=\left(a_{1}, a_{2}, a_{3}\right), b=\left(b_{1}, b_{2}, b_{3}\right)$ and $c=\left(c_{1}, c_{2}, c_{3}\right)$ are boundary (balanced) allocations.

We can find a preference profile $u^{*} \in U$ satisfying the following requirements: (i) for each agent $i=1,2$, agent $i$ 's preference $u_{i}^{*}$ is not differentiable at the quantity $c_{i}$; (ii) agent 3 's preference $u_{3}^{*}$ is not differentiable at the quantity $a_{3}$; (iii) agent 1's preference $u_{1}^{*}$ is differentiable at the quantity $a_{1}$; (iv) the price vector $p$ is a supporting price for $u^{*}$ at $x$, that is, $p \in \Pi\left(x, u^{*}\right)$, and $\Lambda_{i}^{W}(x, p) \subseteq L\left(x_{i}, u_{i}^{*}\right)$ for every agent $i ;(\mathrm{v})$ the price vector $p_{a}=(1 / 4,1 / 2,1 / 4)$ is a supporting price for $u^{*}$ at $a$, that is, $p_{a} \in \Pi\left(a, u^{*}\right)$; (vi) the price vector $p_{b}=(1 / 2,1 / 3,1 / 6)$ is a supporting price for $u^{*}$ at $b$, that is, $p_{b} \in \Pi\left(b, u^{*}\right)$; and (vii) the price vector $p_{c}=(1 / 4,1 / 4,1 / 2)$ is a supporting price for $u^{*}$ at $c$, that 
is, $p_{c} \in \Pi\left(c, u^{*}\right)$.

First, note that the allocation $y$ is a Walrasian allocation at $u^{*}$ with the equilibrium price $p_{y}$ for $y=a, b, c$. Also note that the allocation $x$ is not a Walrasian allocation at $u^{*}{ }^{13}$ Moreover, the set of potential deviators at $(p, x)$ is $N$, that is, $I^{W}(p, x)=N$. Therefore, the hypotheses of part (iv) of condition M-PQ are met. If the Walrasian rule satisfied part (iv) of condition M-PQ, we would have that for some $i$, the pair $\left(p, x_{i}\right)$ would not be a responsible report for $\left(u^{*}, W\right)$. However, since for all $i,\left(p, x_{i}\right)$ is a responsible report for $\left(u^{*}, W\right)$, this is not the case.

Remark 3. We should point out that even though Theorem 2 has been stated and proved assuming that the Walrasian rule is defined on the domain of all profiles of preferences that are continuous, quasi-concave and strongly monotonic on $\mathbb{R}_{+}^{\ell}$ (that is, on $U$ ), its statement continues to hold for any preference domain $U_{N}$ that includes the important special class of all profiles of linear preferences. This change does not harm the proof of Theorem 2 since the fact that the class of linear preferences is a subclass of $U_{N}$ suffices to complete the important step of the actual proof that the set of supporting prices for $u$ at the Walrasian allocation $x$ (at $u$ ) consists solely of Walrasian prices for $u$ (and $W$ ) at $x$; that is, $\pi^{W}(x, u)=$ $\Pi^{W}(x, u)$ for every $u \in U_{N}$ and $x \in W(u)$.

\subsection{On the impossibility of implementing the egalitarian- equivalent and efficient allocation rule}

While the main implication of Theorem 1 is a positive one, as we discussed above, in this subsection we briefly show that condition M-PQ imposes non-trivial restrictions on the class of efficient allocation rules that are semi-responsibly Nash implementable by natural $p-q$ mechanisms.

For an example of an allocation rule that fails to satisfy condition M-PQ, we consider the egalitarian-equivalent and efficient rule, which was introduced by Pazner and Schmeidler (1978).

Egalitarian-equivalent and efficient rule, EE: For each $u \in U, E E(u) \equiv\{x \in$ $A \mid$ there is a maximal number $\lambda \in(0,1)$ such that $u_{i}\left(x_{i}\right)=u_{i}(\lambda \Omega)$ for all $i \in N\} \cap P(u)$, where $U$ is the class of all profiles of utility functions that are continuous, quasi-concave and strongly monotonic on $\mathbb{R}_{+}^{\ell}$.

An allocation is egalitarian-equivalent and efficient in an economy $u$ with respect to a reference bundle if every agent $i$ judges the proposed quantity and a common quantity that is proportional to the aggregate endowment profile as equally good

13. Indeed, noting that $p^{W}$ is the only Walrasian equilibrium price vector whenever $x$ is a Walrasian equilibrium allocation for some economy, $x$ cannot be a Walrasian allocation at $u^{*}$. This is because $x_{1}$ fails to maximize the utility of agent 1 over the budget set defined by the pair $\left(p^{W}, \omega_{1}\right)$ at the economy $u^{*}$, due to the fact that $u_{1}^{*}$ is differentiable at the quantity $x_{1}$ and its gradient vector at $x_{1}$ is equal to $p_{a}$, which is different from $p^{W}$. 
according to $u_{i}$ and if the proposed allocation is efficient for $u$. In other words, every agent obtains the same surplus if we measure the agents' surplus in terms of the reference bundle.

The next result shows that no natural $p-q$ mechanism semi-responsibly Nash implements the egalitarian-equivalent and efficient rule. This is so because this rule fails to satisfy part (iv) of condition M-PQ. Specifically, this rule fails to satisfy our variant of the monotonicity condition due to Saijo, Tatamitani and Yamato (1996, 1999). This result is achieved by restricting the structure of the family $\mathcal{H}$ to the following specification: The family $\mathcal{H}$ has as elements all singletons of the set $N$. This requirement is consistent with Assumption 1 since the mechanism designer cannot exclude any member(s) of the society from being semi-responsible purely on the basis of that assumption.

Claim 1. Let $n \geq 3$. Let Assumption 1 be given. Let the family $\mathcal{H}$ have as elements all singletons of the set $N$. Then, the egalitarian-equivalent and efficient rule does not satisfy condition M-PQ.

Proof. See Appendix.

Remark 4. It is worth emphasizing that the proof of the above negative result is established by employing profiles of utility functions that are strictly concave and differentiable. Thus, the above result continues to hold if we define the egalitarianequivalent and efficient rule on the class of all profiles of utility functions that are strictly concave and differentiable, which is a proper subset of the class $U$.

\section{Concluding remarks}

In this paper, Nash implementation problems by natural $p$ - $q$ mechanisms when agents are semi-responsible are studied. We offer a condition called $\mathbf{M - P Q}$ and show that not only does this condition fully identify the class of efficient rules that are implementable when at least one agent is semi-responsible, but also that the Walrasian rule satisfies it.

Similar to the results of Dutta and Sen (2012) and Kartik et al. (2014), freedisposal plays a crucial role for our results. When feasibility constraints are imposed with an equality, the analysis of implementation problems by natural $p-q$ mechanisms is more difficult, as it requires the construction of an outcome function satisfying the additional property of strong balancedness: assigned quantities must sum to the aggregate endowment for every agents' strategy choices. This analysis is undertaken in Lombardi and Yoshihara (2013b).

In this paper, the mechanism designer knows the individual endowments, but not the individual's preference for quantities. Hurwicz et al. (1995) have shown that the constrained Walrasian rule can be Nash implemented by a $p-q$ mechanism when the mechanism designer knows neither endowments nor preferences. Although the implementation model developed in this paper needs to be modified to handle this 
case, we believe a similar result holds for the Walrasian rule when the mechanism designer knows that at least one agent is semi-responsible, but neither endowments nor preferences. This subject is left for future research.

\section{Appendix}

Proof of Theorem 1. Let the premises hold. Fix any $F \in \mathcal{F}$. The proof that condition M-PQ is necessary for semi-responsible Nash implementation of $F$ by a natural $p-q$ mechanism is discussed in the main text and so is omitted here (see the Addendum below for formal arguments). In the following, we prove that condition M-PQ is also sufficient for it. We then suppose that $F$ satisfies condition M-PQ.

We first establish some notation and definitions. For any $x \in Q^{n}$ and any $p \in P$, define the set $\partial \Lambda_{i}^{F}(x, p)$ by $\partial \Lambda_{i}^{F}(x, p) \equiv\left\{y_{i} \in Q \mid y_{i} \in \Lambda_{i}^{F}(x, p)\right.$ and $\nexists z_{i} \in \Lambda_{i}^{F}(x, p)$ such that $\left.z_{i} \gg y_{i}\right\}$. For each $i \in N$, let $M_{i} \equiv P \times Q$ and let $m_{i}=\left(p^{i}, x_{i}^{i}\right)$ denote agent $i$ 's strategy. For any $m \in M, u \in U_{N}, x \in Q^{n}$ and $p \in P$, define $N_{u}(m)$ by $N_{u}(m) \equiv\left\{i \in N \mid m_{i}=\left(p^{i}, x_{i}^{i}\right)\right.$ is responsible for $\left.(u, F)\right\}$ and $N_{u}(m ;(x, p))$ by $N_{u}(m ;(x, p)) \equiv\left\{i \in N_{u}(m) \mid m_{i}=\left(p, x_{i}\right), x \in F(u), p \in \pi^{F}(x, u)\right\}$. With these preliminaries, we define the outcome function $g$ for any $m \in M$ to be:

Rule 1: If for all $j \in N, m_{j}=\left(p, x_{j}\right), I^{F}(p, x)=N$, then $g(m)=\mathbf{z}(p, x)$.

Rule 2: If for all $j \in N, m_{j}=\left(p, x_{j}\right)$ with $x_{j} \neq 0,1 \leq \# I^{F}(p, x) \leq n-1$, then $g(m)=\overline{\mathbf{z}}(p, x)$.

Rule 3: If for some $i \in N, m_{j}=\left(p, x_{j}\right)$ for all $j \neq i$ and $m_{i}=\left(p^{i}, x_{i}^{i}\right)$, with $p \neq p^{i}$ and $x_{i}^{i} \neq 0, i \in I^{F}(p, x)$, then:

$$
g(m)= \begin{cases}\left(z_{i}\left(\left(p^{i}, x_{i}\right) ;\left(p, x_{-i}\right)\right), 0_{-i}\right) & \text { if } z_{i}\left(\left(p^{i}, x_{i}\right) ;\left(p, x_{-i}\right)\right) \neq 0 \\ \left(x_{i}, 0_{-i}\right) & \text { if } z_{i}\left(\left(p^{i}, x_{i}\right) ;\left(p, x_{-i}\right)\right)=0 \text { and } \\ \left(\hat{x}_{i}, 0_{-i}\right) & x_{i} \in \Lambda_{i}^{F}\left(\left(x_{i}^{\Omega}, x_{-i}\right), p\right) \\ \text { otherwise }\end{cases}
$$

where $\left\{\hat{x}_{i}\right\} \equiv \partial \Lambda_{i}^{F}\left(\left(x_{i}^{\Omega}, x_{-i}\right), p\right) \bigcap\left\{y_{i} \in \mathbb{R}_{+}^{\ell} \mid \exists \alpha \in \mathbb{R}_{+}, y_{i}=\alpha x_{i}\right\}$.

Rule 4: Otherwise:

Rule 4.1: If for some $i, x_{i}^{i}=0, N_{u}(m ;(x, p)) \neq \varnothing$ for some $(u, x, p), \# N_{u}(m ;(x, p)) \geq$ $\# N_{\bar{u}}(m ;(\bar{x}, \bar{p}))$ for all $(\bar{u}, \bar{x}, \bar{p}),{ }^{14}$ then for each $i \in N, g_{i}(m)=\left(\frac{x_{i}}{(n+1)-\# N_{u}(m ;(x, p))}\right)$.

Rule 4.2: Otherwise, $g(m)=0$.

Note that $\gamma \equiv(M, g)$ is a natural $p$ - $q$ mechanism. Note also that in Rule $\mathbf{3}$ agent $i$ can realize any element of $\partial \Lambda_{i}^{F}\left(\left(x_{i}^{\Omega}, x_{-i}\right), p\right)$ by a suitable choice of $m_{i}=\left(p^{i}, x_{i}^{i}\right)$.

14. If there is more than one of type $N_{u}(m ;(x, p))$ having the maximal cardinality, fix any one of them. 
Let $\left(u^{*}, H\right)$ be the true state of the world. We show that $F\left(u^{*}\right)=N A\left(\gamma, R^{\gamma}\left[u^{*}, F, H\right]\right)$. Since it is a routine exercise to prove $F\left(u^{*}\right) \subseteq N A\left(\gamma, R^{\gamma}\left[u^{*}, F, H\right]\right)$, we shall omit the proof here. Conversely, fix any $m \in N E\left(\gamma, R^{\gamma}\left[u^{*}, F, H\right]\right)$. Note, $m$ cannot correspond to Rule 2 nor to Rule 3 nor to Rule $4 .{ }^{15}$ Suppose therefore $m$ falls into Rule 1.

Then, $g(m)=\mathbf{z}(p, x)$. Note that each $i$ can induce Rule $\mathbf{3}$ and attain any quantity $\hat{x}_{i} \in \partial \Lambda_{i}^{F}\left(\left(x_{i}^{\Omega}, x_{-i}\right), p\right)$ by suitably selecting $m_{i}^{\prime}=\left(p^{\prime}, x_{i}^{\prime}\right)$, where $p^{\prime}$ is a boundary point of $P$ and $x_{i}^{\prime} \in Q \backslash \Lambda_{i}^{F}\left(\left(x_{i}^{\Omega}, x_{-i}\right), p\right)$. Then, we have that $\partial \Lambda_{i}^{F}\left(\left(x_{i}^{\Omega}, x_{-i}\right), p\right) \subseteq$ $g_{i}\left(M_{i}, m_{-i}\right)$. It follows from $m \in N E\left(\gamma, R^{\gamma}\left[u^{*}, F, H\right]\right)$ that $\partial \Lambda_{i}^{F}\left(\left(x_{i}^{\Omega}, x_{-i}\right), p\right) \subseteq$ $L\left(\mathbf{z}_{i}(p, x), u_{i}^{*}\right)$. Since $u_{i}^{*}$ is strongly monotonic on $\mathbb{R}_{+}^{\ell}$, it can be seen that $\Lambda_{i}^{F}\left(\left(x_{i}^{\Omega}, x_{-i}\right), p\right) \subseteq$ $L\left(\mathbf{z}_{i}(p, x), u_{i}^{*}\right)$. Note that since $i \in N$ was arbitrary, the latter set inclusion holds for any $i \in N$.

Further, suppose $\mathbf{z}(p, x) \notin F\left(u^{*}\right)$. Condition M-PQ implies that there exists $i \in H$ such that $m_{i}$ is not responsible for $\left(u^{*}, F\right)$ and $u_{i}^{*}\left(z_{i}\left(\left(p^{\prime}, x_{i}^{\prime}\right) ;\left(p, x_{-i}\right)\right)\right)=$ $u_{i}^{*}\left(\mathbf{z}_{i}(p, x)\right)$ for a strategy $\left(p^{\prime}, x_{i}^{\prime}\right)$ that is responsible for $\left(u^{*}, F\right)$. Agent $i$ can change $m_{i}=\left(p, x_{i}\right)$ into $m_{i}^{\prime}=\left(p^{\prime}, x_{i}^{\prime}\right)$. If $p=p^{\prime}$, then she obtains $g_{i}\left(m_{i}^{\prime}, m_{-i}\right)=z_{i}\left(\left(p^{\prime}, x_{i}^{\prime}\right) ;\left(p, x_{-i}\right)\right)$ by either Rule 1 or Rule 2 . On the other hand, if $p \neq p^{\prime}$, then agent $i$ obtains $g_{i}\left(m_{i}^{\prime}, m_{-i}\right)=z_{i}\left(\left(p^{\prime}, x_{i}^{\prime}\right) ;\left(p, x_{-i}\right)\right)$ by Rule 3. In either case, agent $i$ obtains a profitable deviation, in violation of $m \in N E\left(\gamma, R^{\gamma}\left[u^{*}, F, H\right]\right)$. We conclude from this that $\mathbf{z}(p, x) \in F\left(u^{*}\right)$. Since $\left(u^{*}, H\right)$ was arbitrary, the proof is completed.

Proof of Theorem 2. Let the premises hold. Recall Definition 4. Fix any $p \in P$ and $x \in Q^{n}$. Recall $x_{i}^{\Omega}$ denotes $\Omega-\sum_{j \neq i} x_{j}$.

Note that if $x \in W(u)$ for some $u \in U$ and $p \in \pi^{W}(x, u)$, then $p$ is a supporting price for $x$ and for $W$; that is, $p \in \Pi^{W}(x, u)$ (simply because $W$ is defined on $U$ ). Further, if $x \in W(u)$ for some $u \in U$ and $p \in \pi^{W}(x, u)$, then for all $i \in N$, we have $\Lambda_{i}^{W}(x, p)=\left\{y_{i} \in Q \mid p \cdot y_{i} \leq p \cdot \omega_{i}\right\}$. With these preliminaries, let us first verify conditions (i), (ii) and (iii).

Suppose that $I^{W}(p, x)=N$. Define $\mathbf{z}(p, x)$ as follows: (a) $\mathbf{z}(p, x)=x$ if $\sum_{j \in N} x_{j}=\Omega$, and (b) $\mathbf{z}(p, x)=\mathbf{0}$ if $\sum_{j \in N} x_{j} \neq \Omega$. Then for all $i \in N$, we have $\mathbf{z}_{i}(p, x) \in \Lambda_{i}^{W}\left(\left(x_{i}^{\Omega}, x\right), p\right)$. This verifies condition $(i)$.

Suppose that $1 \leq \# I^{W}(p, x) \leq n-1$. Define $\overline{\mathbf{z}}(p, x)$ to be $\overline{\mathbf{z}}(p, x)=\mathbf{0}$. Then, condition (ii) is satisfied.

Fix any $i \in N$. Define $z_{i}\left(\cdot ;\left(p, x_{-i}\right)\right)$ for any $\left(p^{\prime}, x_{i}^{\prime}\right)$ to be (a) $z_{i}\left(\left(p^{\prime}, x_{i}^{\prime}\right) ;\left(p, x_{-i}\right)\right)=$ $x_{i}^{\Omega}$ if $\left(p^{\prime}, x_{i}^{\prime}\right)$ is responsible for some allowable $(u, W), p^{\prime} \neq p$ and $i \in I^{W}\left(p,\left(x_{i}^{\prime}, x_{-i}\right)\right)$; (b) $z_{i}\left(\left(p^{\prime}, x_{i}^{\prime}\right) ;\left(p, x_{-i}\right)\right)=\mathbf{z}_{i}(p, x)$ if $\left(p^{\prime}, x_{i}^{\prime}\right)$ is responsible for some allowable $(u, W)$, $p^{\prime}=p$ and $I^{W}\left(p,\left(x_{i}^{\prime}, x_{-i}\right)\right)=N$; (c) $z_{i}\left(\left(p^{\prime}, x_{i}^{\prime}\right) ;\left(p, x_{-i}\right)\right)=\overline{\mathbf{z}}_{i}(p, x)$ if $\left(p^{\prime}, x_{i}^{\prime}\right)$ is responsible for some allowable $(u, W), p^{\prime}=p, i \in I^{W}\left(p,\left(x_{i}^{\prime}, x_{-i}\right)\right)$ and $1 \leq \# I^{W}\left(p,\left(x_{i}^{\prime}, x_{-i}\right)\right) \leq$ $n-1 ;$ (d) $z_{i}\left(\left(p^{\prime}, x_{i}^{\prime}\right) ;\left(p, x_{-i}\right)\right)=0$, otherwise. We then have condition (iii) satisfied.

Finally, we show that $W$ satisfies condition (iv). Let $I^{W}(p, x)=N$. If $\sum_{j \in N} x_{j} \neq$ $\Omega$, then $\mathbf{z}(p, x)=\mathbf{0}$ and so condition (iv) is vacuously satisfied. Therefore, let $\sum_{j \in N} x_{j}=\Omega$ and so $\mathbf{z}(p, x)=x$. Suppose that for some $u^{*} \in U, \Lambda_{j}^{W}(x, p) \subseteq$ $L\left(x_{j}, u_{j}^{*}\right)$ for all $j \in N$ and $x \notin W\left(u^{*}\right)$. Since $W^{-1}(x, p)$ is non-empty, it follows that 
there is $u \in U$ such that $x \in W(u)$ and $p \in \pi^{W}(x, u)$, and therefore $p \in \Pi^{W}(x, u)$. Since $x \notin W\left(u^{*}\right)$, there is an agent $j \in N$ such that $x_{j} \notin \arg \max _{y_{j} \in \mathbb{R}_{+}^{\ell}: p \cdot y_{j} \leq p \cdot \omega_{j}} u_{j}^{*}\left(y_{j}\right)$. Moreover, since $p \in \pi^{W}(x, u)$ and $u \in U$ imply that $p \gg 0$, there exists $x_{j}^{*}$ such that $x_{j}^{*} \in \arg \max _{y_{j} \in \mathbb{R}_{+}^{\ell}: p \cdot y_{j} \leq p \cdot \omega_{j}} u_{j}^{*}\left(y_{j}\right)$. However, by $\Lambda_{j}^{W}(x, p) \subseteq L\left(x_{j}, u_{j}^{*}\right)$, it follows that $x_{j}^{*} \notin Q$. We then have that $p$ cannot be a competitive equilibrium price for $u^{*}$ and so for all $i \in N,\left(p, x_{i}\right)$ is not responsible for $\left(u^{*}, W\right)$. Let $H \in \mathcal{H}$ and $i \in H$ be given. Let $\left(p^{\prime}, x_{i}^{\prime}\right)$ be any responsible pair for $\left(u^{*}, W\right)$. Then, $p^{\prime} \neq p$. By $I^{W}(p, x)=N$, it follows that $i \in I^{W}\left(p,\left(x_{i}^{\prime}, x_{-i}\right)\right)$. By definition of $z_{i}\left(\cdot ;\left(p, x_{-i}\right)\right)$, we have $z_{i}\left(\left(p^{\prime}, x_{i}^{\prime}\right) ;\left(p, x_{-i}\right)\right)=x_{i}$. This completes the proof of condition (iv).

Proof of Claim 1. Let the premises hold. Take any profile $u \in U$ such that $u_{i}$ is strictly concave and differentiable for every agent $i \in N$. Furthermore, fix any interior allocation $x \in E E(u) \cap \mathbb{R}_{++}^{n \ell}$.

Note that there exists a unique supporting price $p$ for $u$ and $E E$ at $x$, that is, $\pi^{E E}(x, u)=\{p\}$, and that the set $\Lambda_{i}^{E E}(x, p)$ is a well-defined set for every agent $i$. By definition of the rule, it follows that for every $v \in E E^{-1}(x, p)$ it holds that there exists a unique real number $\lambda_{v}$ in the open interval $(0,1)$ such that $v_{i}\left(x_{i}\right)=v_{i}\left(\lambda_{v} \Omega\right)$ for every agent $i$.

Write $\lambda_{\min }$ for the solution to the problem:

$$
\min \left\{\lambda_{v} \mid \text { for } v \in E E^{-1}(x, p): v_{i}\left(x_{i}\right)=v_{i}\left(\lambda_{v} \Omega\right) \text { for every } i \in N\right\},
$$

and write $\partial \Lambda_{i}^{E E}(x, p)$ for the upper boundary of $\Lambda_{i}^{E E}(x, p)$, that is:

$$
\partial \Lambda_{i}^{E E}(x, p) \equiv\left\{y_{i} \in Q \mid y_{i} \in \Lambda_{i}^{E E}(x, p) \text { and } \nexists z_{i} \in \Lambda_{i}^{E E}(x, p) \text { such that } z_{i} \gg y_{i}\right\} \text {. }
$$

By construction, it follows that the common quantity $\lambda_{\min } \Omega$ is an element of $\partial \Lambda_{i}^{E E}(x, p)$ for every agent $i$. Moreover, for every agent $i$ it also holds that there is a proper subset $B\left(x_{i}\right)$ of $\partial \Lambda_{i}^{E E}(x, p)$, which constitutes a neighborhood of $x_{i}$ in $\partial \Lambda_{i}^{E E}(x, p)$. By taking such an neighborhood sufficiently small, it follows from the definition of $\partial \Lambda_{i}^{E E}(x, p)$ that $p \cdot y_{i}=p \cdot x_{i}$ for every quantity $y_{i} \in B\left(x_{i}\right)$, since $x_{i}$ is an interior consumption bundle.

Fix any two distinct agents $j$ and $k$. Take any profile $u^{*} \in U$ such that $u_{i}^{*}$ is strictly concave and differentiable for every agent $i$, that $\Lambda_{i}^{E E}(x, p) \subseteq L\left(x_{i}, u_{i}^{*}\right)$ for every agent $i$ and that for a sufficiently small real number $\epsilon>0$ and for a real number $\lambda>\lambda_{\min }$ it holds that:

$$
\begin{aligned}
u_{i}^{*}\left(x_{i}\right) & =u_{i}^{*}(\lambda \Omega) \text { for every agent } i \neq j, k, \\
u_{j}^{*}\left(x_{j}+(\epsilon, 0)\right) & =u_{j}^{*}(\lambda \Omega), \\
u_{k}^{*}\left(x_{k}-(\epsilon, 0)\right) & =u_{k}^{*}(\lambda \Omega) \text { and } \\
D u_{j}^{*}\left(x_{j}+(\epsilon, 0)\right) & =\{p\}=D u_{k}^{*}\left(x_{k}-(\epsilon, 0)\right),
\end{aligned}
$$

where 0 is the $\ell-1$-th dimensional zero vector and where $D u_{j}^{*}\left(x_{j}+(\epsilon, 0)\right)$ and $D u_{k}^{*}\left(x_{k}-(\epsilon, 0)\right)$ denote the gradient vector respectively at the quantity $x_{j}+(\epsilon, 0)$ and at $x_{k}-(\epsilon, 0)$. This profile $u^{*}$ exists because of our domain assumption. By 
the construction of $u^{*}, B\left(x_{i}\right) \subseteq L\left(x_{i}, u_{i}^{*}\right)$ for every agent $i$, which implies that $\{p\}=\Pi\left(x, u^{*}\right)$. Thus, $x \in P O\left(u^{*}\right)$.

By the above discussion, there exist a real number $\lambda_{j}$ in the open interval $\left(\lambda_{\min }, \lambda\right)$ and a real number $\lambda_{k}>\lambda$ such that $u_{j}^{*}\left(x_{j}\right)=u_{j}^{*}\left(\lambda_{j} \Omega\right)$ and that $u_{k}^{*}\left(x_{k}\right)=u_{k}^{*}\left(\lambda_{k} \Omega\right)$. Therefore, since $\lambda_{k}>\lambda>\lambda_{j}, x$ is not egalitarian-equivalent at the profile $u^{*}$. Thus, $x \in P O\left(u^{*}\right) \backslash E E\left(u^{*}\right)$. The allocation $x^{*}$, which assigns the quantity $x_{i}^{*}=x_{i}$ to every agent $i \neq j, k$, the quantity $x_{j}^{*}=x_{j}+(\epsilon, 0)$ to agent $j$ and the quantity $x_{k}^{*}=x_{k}-(\epsilon, 0)$ to agent $k$, is an egalitarian-equivalent and efficient allocation at $u^{*}$ and, moreover, the price vector $p$ is a supporting price for $u^{*}$ and possibly for $E E$ at this $x^{*}$, that is, $p \in \pi^{E E}\left(x^{*}, u^{*}\right){ }^{16}$

Take any agent $i \neq j, k$. The singleton $\{i\}$ is an element of the collection $\mathcal{H}$ since this collection has as elements all singletons of the set $N$ and since, moreover, there are $n \geq 3$ agents in $N$. We have that the pair $\left(p, x_{i}\right)$ is responsible for $\left(u^{*}, E E\right)$, in violation of part (iv) of condition M-PQ.

\section{References}

Corchón L, Herrero C (2004) A decent proposal. Span Econ Rev 6:107-125

Bochet O (2007) Implementation of the Walrasian Correspondence: the Boundary Problem. Int J Game Theory 36:301-316 (2007)

Doğan B (2014) Essays on Mechanism Design and Implementation, PhD dissertation, University of Rochester

Dutta B, Sen A (2012) Nash implementation with partially honest individuals. Games Econ Behav 74:154-169

Dutta B, Sen A, Vohra R (1995) Nash implementation through elementary mechanisms in economic environments. Econ Des 1:173-204

Eliaz K (2002) Fault-tolerant implementation. Rev Econ Stud 69:589-610

Glazer J, Rubinstein A (1998) Motives and implementation: On the design of mechanisms to elicit opinions. J Econ Theory 79:157-173

Hurwicz L (1978) On the interaction between information and incentives in organizations. In Krippendorff K (eds) Communication and Control in Society, 123-147. Scientific Publishers, Inc., New York

Hurwicz L, Maskin E, and Postlewaite A (1995) Feasible Nash implementation of social choice rules when the designer does not know endowments or production sets. In Ledyard $\mathrm{J}$ (eds) The economics of informational decentralization: Complexity, efficiency, and stability, Essays in Honor of Stanley Reiter, 367-433. Kluwer, Amsterdam Jackson MO (1992) Implementation in undominated strategies: A look at bounded mechanisms. Rev Econ Stud 59:757-775

16. Note that since the egalitarian-equivalent and efficient rule is essentially single-valued, and since, moreover, each $u_{i}^{*}$ is strictly concave, it follows that $E E\left(u^{*}\right)=\left\{x^{*}\right\}$. An allocation rule $F$ is essentially single-valued if for every allowable profile $u$ it holds that: $x, x^{\prime} \in F(u) \Longrightarrow u_{i}\left(x_{i}\right)=$ $u_{i}\left(x_{i}^{\prime}\right)$ for every agent $i \in N$. 
Jackson MO (2001) A crash course in implementation theory. Soc Choice Welfare 18:655-708

Kartik N, Tercieux O (2012) Implementation with evidence. Theoretical Econ 7:323355

Kartik N, Tercieux O, Holden R (2014) Simple mechanisms and preferences for honesty. Games Econ Behav 83:284-290

Lombardi M, Yoshihara N (2013a) A full characterization of Nash implementation with strategy space reduction. Econ. Theory 54:131-151

Lombardi M, Yoshihara N (2013b) Natural implementation with partially honest agents. IER Discussion Paper Series A, No.561, The Institute of Economic Research, Hitotsubashi University

Lombardi M, Yoshihara N (2014) Natural implementation with partially-honest agents in economic environments with free-disposal. IER Discussion Paper Series A, No.616, The Institute of Economic Research, Hitotsubashi University

Maskin E (1999) Nash equilibrium and welfare optimality. Rev Econ Stud 66:23-38

Matsushima H (2008a) Role of honesty in full implementation. J Econ Theory 139:353-359

Matsushima H (2008b) Behavioral aspects of implementation theory. Econ Lett 100:161-164

Otani Y, Sicilian J (1982) Equilibrium of Walras preference games. J Econ Theory 27:47-68

Pazner E, Schmeidler D (1978) Egalitarian Equivalent Allocations: A New Concept of Economic Equity. QJ Econ 92:671-686.

Postlewaite A, Wettstein D (1989) Feasible and Continuous Implementation. Rev Econ Stud 56:603-611

Saijo T, Tatamitani Y, Yamato T (1996) Toward natural implementation. Int Econ Rev 37:949-980

Saijo T, Tatamitani Y, Yamato T (1999) Characterizing natural implementability: The fair and Walrasian correspondences. Games Econ Behav 28:271-293

Saporiti A (2014) Securely implementable social choice rules with partially honest agents. J Econ Theory 154:216-228

Sjöström T (1996) Implementation by demand mechanisms. Econ Des 1:343-354

Tatamitani Y (2001) Implementation by self-relevant mechanisms. J Math Econ $35: 427-444$

Thomson W (1984) The manipulability of resource allocation mechanisms. Rev Econ Stud 51:447-460

Thomson W (1999) Monotonic extensions on economic domains. Rev Econ Design 4:13-33

Tian G (1992) Implementation of the Walrasian Correspondence without Continuous, Convex, and Ordered Preferences. Soc. Choice Welfare 9:117-130 